\title{
Efecto de la pasteurización en la concentración de diclorodifeniltricloroetano (DDT) y hexaclorociclohexano (HCH)
}

\author{
en leche de bovino
}

Violeta Trinidad Pardío Sedas ${ }^{\text {a }}$

Karla María López Hernández ${ }^{\mathrm{a}^{*}}$

Argel Flores Primo ${ }^{a}$

Roxana Uscanga Serrano ${ }^{\text {a }}$

a Universidad Veracruzana. Facultad de Medicina Veterinaria y Zootecnia, Av. Miguel Ángel de Quevedo s/n, Col. Unidad Veracruzana, 91710. Veracruz, Veracruz, México.

*Autor de correspondencia: klopez@uv.mx

\section{Resumen:}

El diclorodifeniltricloroetano (DDT) y hexaclorociclohexano (HCH) son disruptores endocrinos cuya presencia en la leche representa un riesgo para la salud. Existe evidencia de que la pasteurización disminuye o incrementa la concentración de plaguicidas organoclorados en productos lácteos. La presente investigación evaluó el efecto de la pasteurización a $63{ }^{\circ} \mathrm{C} 30 \mathrm{~min}^{-1}$ y $73{ }^{\circ} \mathrm{C} 15 \mathrm{seg}^{-1}$ en las concentraciones de DDT, HCH y sus metabolitos en leche bovina, para estimar la exposición dietaria por consumo humano de leche pasteurizada. Se realizó un diseño experimental completamente al azar con dos tratamientos en 100 muestras de leche recolectadas en Soledad de Doblado y Jamapa, Veracruz, México. Los plaguicidas se cuantificaron por cromatografía de gases con detector de microcaptura de electrones. Los datos se analizaron mediante análisis de varianza unifactorial $(P<0.05)$ y las medias se compararon con Tukey $(P<0.05)$. La exposición dietaria a plaguicidas se evaluó por la ingesta diaria estimada (IDE) y dosis diaria promedio (DDP) en tres grupos de población. La pasteurización a $73^{\circ} \mathrm{C}$ disminuyó 30.94, 44.51, 3.18, 81.23 y $42.82 \%$ las concentraciones de p,p'-DDE, p,p'-DDD, o,p'-DDT, p,p'-DDT y DDT 
total, respectivamente, así como las concentraciones de $\beta-\mathrm{HCH}, \gamma-\mathrm{HCH}$ y $\mathrm{HCH}$ total (85.68, 18.88 y $99.31 \%$, respectivamente). La IDE para niños, adultos y ancianos de DDT total fue menor por consumo de leche pasteurizada a $73{ }^{\circ} \mathrm{C}$ y de $\gamma-\mathrm{HCH}$ a $63{ }^{\circ} \mathrm{C}$. La DDP de DDT total disminuyó con la pasteurización a $73{ }^{\circ} \mathrm{C}$. La exposición dietaria de DDT y HCH fue mayor en niños.

Palabras clave: DDT, HCH, Leche, Pasteurización, Exposición dietaria, Niños.

Recibido: 22/08/2019

Aceptado: 02/07/2020

\section{Introducción}

Los plaguicidas organoclorados (POC), como diclorodifeniltricloroetano (DDT) y hexaclorociclohexano $(\mathrm{HCH})$, se usaron abundantemente en todo el mundo a partir de 1949, principalmente en programas de agricultura y salud pública para la prevención de plagas, malezas y otros patógenos en países tropicales ${ }^{(1)}$. En México, el DDT fue el insecticida utilizado para el control de la malaria; en Veracruz se aplicó rutinariamente hasta el 2003 en regiones endémicas y el $\gamma$-HCH (lindano) se utilizó para el control de plagas veterinarias ${ }^{(2)}$, ya que este isómero es considerado el elemento activo del $\mathrm{HCH}$ técnico con propiedades insecticidas específicas ${ }^{(3,4)}$. En mayo de 2004 quedó restringido el uso de estos plaguicidas después de entrar en vigor el Convenio de Estocolmo. Este tratado internacional tuvo el objeto de proteger la salud humana y el ambiente de sustancias químicas tóxicas, persistentes y bioacumulables ${ }^{(5)}$. Sin embargo, en México y otros países el lindano se usa como conservador de semillas, como ectoparasiticida en ganadería y en lociones o jabones para tratamiento de sarnas y piojos en humanos ${ }^{(6)}$. Los POC son compuestos que, por su presión de vapor y coeficiente de partición, son persistentes y móviles en el medio ambiente ${ }^{(7)}$ y por su naturaleza lipófila se bioacumulan y biomagnifican a través de la cadena alimentaria ${ }^{(8,9)}$. El DDT y HCH son considerados compuestos cancerígenos y disruptores endocrinos ${ }^{(4,10)}$ y sus residuos han sido reportados en animales y humanos ${ }^{(11)}$. La vida media de los POC puede oscilar entre algunos meses y varios años hasta décadas. Se ha estimado que la degradación del DDT en el suelo varía de 4 a 30 años ${ }^{(12)}$.

La exposición de los animales al DDT y HCH puede aumentar a partir del tratamiento directo con plaguicidas, la inhalación de aire o la ingestión de forrajes y piensos contaminados ${ }^{(2)}$. En los organismos bovinos después de la reabsorción, los POC ingresan al hígado y se metabolizan lentamente antes de que se liberen en el sistema circulatorio y finalmente se depositan en la grasa o se eliminan a través de la leche, pasando al ternero o al consumidor humano $^{(9,13)}$. Diversos estudios han demostrado la presencia de DDT y HCH en leche, carne y tejidos de bovino ${ }^{(9,14,15)}$. Más aún, la leche bovina se ha utilizado como un indicador de la 
persistencia de estos plaguicidas debido a la alimentación animal, inhalación del aire y el uso intensivo en programas para el control de ectoparásitos en el ganado. En este contexto, se han realizado monitoreos de los niveles de POC en estos alimentos para estimar la exposición de la población y los posibles riesgos para la salud ${ }^{(16,17)}$.

La producción nacional de leche bovina en 2018 fue de 11 mil 923 millones de litros, de los cuales el estado de Veracruz aportó el 6.0 \% de la producción. México ocupó el octavo lugar en producción lechera a nivel mundial, destacando que la Unión Europea, considerada como entidad geopolítica conformada por 28 países, ocupó el primer lugar de producción de leche ${ }^{(18,19)}$. En México el consumo per cápita de leche durante 2018 fue de 339 $\mathrm{ml} /$ persona/día ${ }^{(18)}$. En base a su composición, la leche es un alimento completo y equilibrado que proporciona un elevado contenido de nutrientes en relación con su contenido calórico, por lo que su consumo debe considerarse necesario desde la infancia a la tercera edad ${ }^{(20)}$. Debido a la importancia que tiene este producto como alimento, se han desarrollado varias investigaciones con el propósito de reducir el contenido de los POC en la leche. Abd-Rabo et $a l^{(21)}$ reportaron que la pasteurización de leche de búfala a $73^{\circ} \mathrm{C}$ ocasionó una disminución del $23.07 \%$ de la concentración inicial de p,p'-DDE y un $32.85 \%$ de p,p'-DDT, pero un incremento del $30 \%$ en la concentración de p,p'-DDD. Deiana y Fatichenti ${ }^{(22)}$ reportaron un incremento del $6.5 \%$ en la concentración de DDT y HCH en leche de bovino pasteurizada a $73{ }^{\circ} \mathrm{C}$. Sin embargo, Abou-Arab ${ }^{(23)}$ observó la disminución de $\gamma$-HCH $(72.90$ y $65.00 \%)$ en leche de bovino pasterizada a 63 y $72{ }^{\circ} \mathrm{C}$.

No obstante, son pocas las investigaciones que evalúan el riesgo a la salud asociado al consumo de leche bronca y pasteurizada comercializada contaminada con estos plaguicidas ${ }^{(24,25)}$. Abou-Arab et $a l^{(26)}$ encontraron que la ingesta diaria estimada (IDE) de $\gamma$ $\mathrm{HCH}$ en leche bronca, pasteurizada y ultrapasteurizada fue $0.28,0.11$ y $0.03 \mu \mathrm{g} \mathrm{kg}^{-1} \mathrm{pc}^{-}$día $^{-}$ ${ }^{1}$, respectivamente; a su vez, el metabolito del DDT con mayor IDE fue o,p'-DDE $(0.48 \mu \mathrm{g}$ $\mathrm{kg}^{-1} \mathrm{pc}$ día $\left.{ }^{-1}\right)$ por consumo de leche bronca y o,p'-DDD $\left(0.60 \mu \mathrm{g} \mathrm{kg}^{-1} \mathrm{pc}_{\text {día }}^{-1}\right)$ por consumo de leche pasterizada. Amir et al ${ }^{(17)}$ evaluaron la presencia de POC en diferentes alimentos, pero las IDEs más altas correspondieron a la leche bronca con $0.011,0.074$ y $0.103 \mu \mathrm{g} \mathrm{kg}^{-1}$ pc día ${ }^{-1}$ para $\gamma-\mathrm{HCH}, \mathrm{HCH}$ total y DDT total, respectivamente. Miclean et al ${ }^{(27)}$ reportaron IDE de HCH total para mujeres, hombres y niños de $0.002,0.002$ y $0.012 \mu \mathrm{g} \mathrm{kg}^{-1} \mathrm{pc} \mathrm{día}^{-1}$, respectivamente, y de DDT total $0.001,0.002$ y $0.008 \mu \mathrm{g} \mathrm{kg}^{-1} \mathrm{pc} \mathrm{día}^{-1}$, respectivamente.

En base a lo anterior, el objetivo de esta investigación fue determinar el efecto de la pasteurización a $63{ }^{\circ} \mathrm{C} 30 \mathrm{~min}^{-1}$ (lenta de baja temperatura) y $73{ }^{\circ} \mathrm{C} 15 \mathrm{seg}^{-1}$ (rápida de alta temperatura) en los niveles de concentración de DDT, $\mathrm{HCH}$ y sus metabolitos (p,p'-DDE, p,p'-DDD, o,p'-DDT, p,p'-DDT, $\alpha-\mathrm{HCH}, \beta \mathrm{HCH}, \gamma-\mathrm{HCH}$ y $\delta$-HCH) en leche bovina procedente de la zona agraria central del Estado de Veracruz, México y estimar la exposición 
dietaria de grupos de población de niños, adultos y ancianos a estos plaguicidas contenidos en leche bronca y pasteurizada a estas dos temperaturas, mediante la IDE y la DDP.

\section{Material y métodos}

Se seleccionaron 10 animales clínicamente sanos de más de dos años, con más de un parto que estuvieron en ordeña en dos unidades de producción (UP) ubicadas en el municipio de Soledad de Doblado (1903' N, 96 24' O) y Jamapa (1901' N, 96 $13^{\circ}$ ' O), Veracruz, México. Los municipios se caracterizan por tener clima cálido subhúmedo con lluvia en verano, el rango anual de precipitación es de 900 a 1,100 mm y 1,100 a 1,300, respectivamente, el rango anual de temperatura es de 24 a $26^{\circ} \mathrm{C}^{(28,29)}$. El estudio se realizó con la aprobación del Comité de Bioética y Bienestar Animal de la Facultad de Medicina Veterinaria y Zootecnia. Se recolectaron mensualmente durante un ciclo anual (2017-2018) un total de 100 muestras de leche bovina (de $500 \mathrm{ml}$ cada una) de la primera ordeña de los mismos animales seleccionados y se transportaron al Laboratorio de Toxicología de la Facultad de Medicina Veterinaria y Zootecnia de la Universidad Veracruzana de acuerdo con la NOM-243-SSA1$2010^{(30)}$. En cada muestreo se realizó un pool de las muestras recolectadas procedentes de ambas UP del cual la mitad de las muestras se asignaron aleatoriamente a la pasteurización lenta y el resto a la pasteurización rápida. De tal forma que, de las 100 muestras, 25 muestras se pasteurizaron a $63{ }^{\circ} \mathrm{C} 30 \mathrm{~min}^{-1}$ y otras 25 muestras sin pasteurizar correspondieron al testigo de $63{ }^{\circ} \mathrm{C}$. Así mismo, 25 muestras se pasteurizaron a $73{ }^{\circ} \mathrm{C} 15 \mathrm{seg}^{-1}$ y otras 25 muestras sin pasteurizar correspondieron al testigo de $73{ }^{\circ} \mathrm{C}$. De esta forma, se simuló la mezcla de la leche bronca de diferente procedencia en los centros de acopio y que es vendida a la industria láctea en la zona.

\section{Pasteurización de la leche}

El proceso de pasteurización se realizó en el Laboratorio de Toxicología en base a la norma NOM-243-SSA1-2010(30). Brevemente: $500 \mathrm{ml}$ de leche bronca se calentaron para la pasteurización lenta a $63{ }^{\circ} \mathrm{C}$ durante $30 \mathrm{~min}$ y $500 \mathrm{ml}$ para la pasteurización rápida a $73{ }^{\circ} \mathrm{C}$ durante $15 \mathrm{seg}$; al término de cada pasteurización, las muestras se enfriaron rápidamente a $4{ }^{\circ} \mathrm{C}$ y, una vez alcanzada esta temperatura, inmediatamente se centrifugaron a 3,500 rpm para separar la grasa, la cual se almacenó en viales color ámbar etiquetados y se almacenaron a $-20{ }^{\circ} \mathrm{C}$ hasta el momento de su análisis.

\section{Determinaciones analíticas}

La determinación del contenido etéreo de la leche se realizó por el método de Gerber ${ }^{(31)}$ y la determinación de los niveles de concentración de DDT, HCH y sus metabolitos en la grasa de la leche se realizó de acuerdo con la metodología modificada de Murphy ${ }^{(32)}$. Todos los 
productos químicos empleados en los análisis fueron grado analítico de marca Merck (Darmstadt, Alemania), J.T. Baker y Sigma-Aldrich Company (St. Louis, MO, EE. UU.). Cada muestra se analizó por triplicado y los resultados se expresaron como $\mu \mathrm{g} \mathrm{kg}^{-1}$ base lipídica. Los residuos químicos de los análisis almacenados en galones ámbar fueron recolectados por la compañía EcoEntorno S.A. de C.V. contratada por la Universidad Veracruzana para la colecta de residuos peligrosos.

\section{Análisis cromatográfico}

La concentración de DDT, HCH y sus metabolitos se cuantificaron en un cromatógrafo de gases Agilent-Hewlett-Packard 6890 Plus con automuestreador 7683 y con un detector de microcaptura de electrones con fuente Ni-63. Las condiciones de operación fueron las siguientes: inyector en modo splitless, flujo de purga de $60 \mathrm{ml} \mathrm{min}^{-1}$, tiempo de purga 0.80 min y temperatura de $250{ }^{\circ} \mathrm{C}$, columna HP-608 de $30 \mathrm{~m}$ de largo x $530 \mu \mathrm{m}$ de diámetro y 0.5 $\mu \mathrm{m}$ de grosor de película, con flujo constante de nitrógeno ultrapuro de $2 \mathrm{ml} \mathrm{min}^{-1}$. Se programó el horno con la siguiente rampa de temperatura: temperatura inicial de $80{ }^{\circ} \mathrm{C}$ a $180{ }^{\circ} \mathrm{C}$ a $30^{\circ} \mathrm{C} \mathrm{min}^{-1}$, y de $280^{\circ} \mathrm{C}$ a $10^{\circ} \mathrm{C} \mathrm{min}^{-1}$ sostenida $2.7 \mathrm{~min}$. El tiempo de corrida fue $19.03 \mathrm{~min}$, la temperatura del detector de $320^{\circ} \mathrm{C}$ con flujo constante de nitrógeno de $30 \mathrm{ml}$ $\min ^{-1} \mathrm{y}$ el volumen de inyección $1 \mu \mathrm{l}$.

\section{Linearidad, límites de detección y cuantificación}

La linearidad del detector se determinó mediante análisis de regresión linear efectuado por el software ChemStation a partir de cinco puntos en las curvas de calibración para cada plaguicida. La calibración se realizó previamente al análisis de las muestras utilizando los estándares de los POC adquiridos en ChemService (Chem Service, Inc., West Chester, PA, USA) y Supelco (Supelco Park, Bellefonte, PA, USA). El análisis cualitativo y cuantitativo se efectuó mediante la comparación de los tiempos de retención y el área del pico de la muestra, respectivamente, con los estándares de referencia de la calibración. El límite de detección (LOD) y de cuantificación (LOQ) se calculó para cada plaguicida de acuerdo a $\mathrm{Su}^{(33)}$. Para validar dicho método se usaron muestras de leche fortificadas con una recuperación de 91 a $99 \%$. El LOD para DDT y HCH varió de 0.0004-0.00036 y 0.00002$0.00031 \mu \mathrm{g} \mathrm{kg}^{-1}$, respectivamente y el LOQ varió de $0.001 \mu \mathrm{g} \mathrm{kg}^{-1}$ base lipídica.

\section{Valoración de la exposición dietaria a los plaguicidas}

La exposición dietaria a DDT, HCH y sus metabolitos se evaluó mediante la ingesta diaria estimada (IDE) y la dosis diaria promedio (DDP) de acuerdo a Pandit y Sahu ${ }^{(34)}$. El riesgo se estimó en tres grupos de población, niños, adultos y ancianos. La IDE se reportó en $\mu \mathrm{g}$ de plaguicida $\mathrm{kg}^{-1}$ pc (peso corporal) día ${ }^{-1}$ y se calculó con la siguiente fórmula: $I D E=C_{a} \times$ 
$F \times I$; donde $\mathrm{C}_{a}$ : nivel medio de residuos de plaguicidas organoclorados en muestras de leche ( $\mu \mathrm{g} \mathrm{kg}^{-1}$ base lipídica), F: contenido de grasa en muestras de leche (\%), I: ingesta de leche en mililitros por kilos de peso corporal por día $\left(\mathrm{mlkg}^{-1} \mathrm{pc} \mathrm{día}^{-1}\right)$. La ingesta diaria de leche (339 $\mathrm{ml}$ por persona día ${ }^{-1}$ ) utilizada para calcular las IDE fue considerada en base al consumo per cápita de leche en México durante 2018, y los pesos promedio por persona fueron: $25 \mathrm{~kg}$ en niños ${ }^{(35)}, 70 \mathrm{~kg}$ en adultos $^{(36)}$ y $51 \mathrm{~kg}$ en ancianos ${ }^{(37)}$.

La dosis diaria promedio (DDP) es la tasa de dosis promedio en un periodo específico de exposición expresada en unidades de masa-tiempo $\left(\mu \mathrm{g} \mathrm{kg}^{-1} \mathrm{dí}^{-1}\right)$ calculada con la siguiente fórmula: $D D P=C_{m} \times F \times I$; donde $\mathrm{C}_{\mathrm{m}}$ : concentración máxima de plaguicidas organoclorados en muestras de leche $\left(\mu \mathrm{g} \mathrm{kg}^{-1}\right), \mathrm{F}$ : contenido de grasa en muestras de leche (\%), I: ingesta de leche ( $\left.\mathrm{ml} \mathrm{kg}^{-1} \mathrm{pc} \mathrm{día}^{-1}\right)$. Para la DDP de plaguicidas se consideró la siguiente ingesta diaria de leche $(\mathrm{ml})$ y pesos recomendados $(\mathrm{kg})$ en tres grupos principales de población: niños $(480 \mathrm{ml} / 25 \mathrm{~kg})^{(35)}$, adultos $(240 \mathrm{ml} / 70 \mathrm{~kg})^{(38)} \mathrm{y}$ ancianos $(310 \mathrm{ml} / 51 \mathrm{~kg})^{(37)}$.

\section{Análisis estadístico}

Se empleó un diseño experimental completamente al azar, siendo la fuente de variación el método de pasteurización lenta y rápida de la leche para evaluar su efecto en los niveles de concentración de DDT, HCH y sus metabolitos (p,p'-DDE, p,p'-DDD, o,p'-DDT, p,p'-DDT, $\alpha-\mathrm{HCH}, \beta-\mathrm{HCH}, \gamma-\mathrm{HCH}$ y $\delta-\mathrm{HCH})$ en la leche. El modelo estadístico del diseño experimental fue el siguiente:

$\mathrm{Y}_{\mathrm{ij}}=\mu+\tau_{\mathrm{i}}+\varepsilon_{\mathrm{ij}}$

En donde:

$\boldsymbol{Y}_{i j}$ es la variable de respuesta (concentración de los POCs) de la ij-ésima unidad experimental (cada muestra de leche) (i y j denotan el nivel del factor y la replicación en el nivel del factor, respectivamente);

$\boldsymbol{\mu}$ es el efecto de la media general;

$\tau_{i}$ es el efecto del i-ésimo tratamiento (pasteurización lenta y rápida);

$\varepsilon_{i j}$ es el efecto del error experimental asociado a la i-ésima unidad experimental.

Con las concentraciones obtenidas se realizó un ANDEVA de una vía $(P<0.05)$ para evaluar el efecto de la pasteurización a 63 y $73{ }^{\circ} \mathrm{C}$ en los niveles medios de concentración de DDT, $\mathrm{HCH}$ y sus metabolitos en la leche de bovino bronca. Las diferencias significativas entre las medias se determinaron por prueba de Tukey $(P<0.05)$ mediante el programa estadístico Minitab v.17.0. 


\section{Resultados y discusión}

\section{Niveles de concentración de DDT y HCH en leche bronca}

De acuerdo a los resultados (Cuadro 1), las concentraciones de p,p'-DDE, p,p'-DDD, o,p'DDT y DDT total $(15.736 \pm 8.133,9.849 \pm 5.271,15.237 \pm 9.006$ y $26.480 \pm 13.880 \mu \mathrm{g}$ $\mathrm{kg}^{-1}$ base lipídica, respectivamente) de la leche bronca utilizada como control de la pasteurización a $63^{\circ} \mathrm{C}$ fueron más altas $(P<0.05)$ con respecto a la leche control pasteurizada a $73{ }^{\circ} \mathrm{C}\left(3.829 \pm 2.172,4.904 \pm 3.493,3.734 \pm 1.526\right.$ y $8.920 \pm 7.340 \mu \mathrm{g} \mathrm{kg}^{-1}$ base lipídica, respectivamente). Sin embargo, las concentraciones de DDT total en las leches testigo no rebasaron el Límite Máximo de Residuos (LMR) de $50 \mu \mathrm{g} \mathrm{kg}^{-1}$ (base lipídica) establecido por la Food and Agricultural Organization/World Health Organization ${ }^{(39)}$.

Cuadro 1: Media y desviación estándar de DDT, $\mathrm{HCH}$ y sus metabolitos $\left(\mu \mathrm{g} \mathrm{kg}^{-1}\right.$ base lipídica) en leche bronca control y pasteurizada

\begin{tabular}{|c|c|c|c|c|}
\hline & \multicolumn{2}{|c|}{ Tratamiento de $63^{\circ} \mathrm{C} 30 \mathrm{~min}^{-1}$} & \multicolumn{2}{|c|}{ Tratamiento de $73{ }^{\circ} \mathrm{C} 15 \mathrm{seg}^{-1}$} \\
\hline & $\begin{array}{l}\text { Leche } \\
\text { control } \\
(\mathbf{n}=\mathbf{2 5})\end{array}$ & $\begin{array}{l}\text { Leche } \\
\text { pasteurizada } \\
(\mathrm{n}=25)\end{array}$ & $\begin{array}{l}\text { Leche } \\
\text { control } \\
(\mathbf{n}=\mathbf{2 5})\end{array}$ & $\begin{array}{l}\text { Leche } \\
\text { pasteurizada } \\
(\mathbf{n}=\mathbf{2 5})\end{array}$ \\
\hline p,p'-DDE & $15.736 \pm 8.133^{\mathrm{ax}}$ & $10.673 \pm 5.682^{\mathrm{b}}$ & $3.829 \pm 2.172^{\mathrm{ay}}$ & $2.644 \pm 2.023^{\mathrm{a}}$ \\
\hline p,p'-DDD & $9.849 \pm 5.271^{\mathrm{ax}}$ & $8.334 \pm 4.771^{\mathrm{a}}$ & $4.904 \pm 3.493^{\text {ay }}$ & $2.721 \pm 1.333^{\mathrm{a}}$ \\
\hline o,p'-DDT & $15.237 \pm 9.006^{\mathrm{ax}}$ & $7.559 \pm 4.015^{\mathrm{b}}$ & $3.734 \pm 1.526^{\text {ay }}$ & $3.615 \pm 1.831^{\mathrm{a}}$ \\
\hline $\mathrm{p}, \mathrm{p}^{\prime}-\mathrm{DDT}$ & $13.441 \pm 7.905^{\mathrm{ax}}$ & $13.484 \pm 6.673^{\mathrm{a}}$ & $11.351 \pm 6.485^{\mathrm{ax}}$ & $2.130 \pm 1.566^{\mathrm{a}}$ \\
\hline DDT total & $\begin{array}{l}26.480 \pm \\
13.880^{\mathrm{ax}}\end{array}$ & $20.170 \pm 16.05^{\mathrm{a}}$ & $8.920 \pm 7.340^{\text {ay }}$ & $5.100 \pm 3.210^{\mathrm{a}}$ \\
\hline$\alpha-\mathrm{HCH}$ & $0.270 \pm 0.154^{\mathrm{ax}}$ & $0.355 \pm 0.137^{b}$ & $0.310 \pm 0.111^{\mathrm{ax}}$ & $0.543 \pm 0.151^{b}$ \\
\hline$\beta-\mathrm{HCH}$ & $0.401 \pm 0.276^{\mathrm{ax}}$ & $0.919 \pm 0.062^{\mathrm{b}}$ & $1.438 \pm 0.376^{\text {ay }}$ & $0.206 \pm 0.113^{b}$ \\
\hline$\gamma-\mathrm{HCH}$ & $0.511 \pm 0.338^{\mathrm{ax}}$ & $0.702 \pm 0.236^{\mathrm{a}}$ & $1.187 \pm 0.204^{\mathrm{ay}}$ & $0.963 \pm 0.122^{\mathrm{a}}$ \\
\hline$\delta-\mathrm{HCH}$ & $0.424 \pm 0.229^{\mathrm{ax}}$ & $1.504 \pm 2.379^{\mathrm{a}}$ & $0.523 \pm 0.346^{\mathrm{ax}}$ & $1.504 \pm 2.379^{\mathrm{a}}$ \\
\hline $\mathrm{HCH}$ total & $0.552 \pm 0.368^{\mathrm{ax}}$ & $1.067 \pm 1.076^{\mathrm{a}}$ & $1.241 \pm 0.830^{\mathrm{ax}}$ & $0.684 \pm 0.452^{\mathrm{a}}$ \\
\hline $\begin{array}{l}\text { DDT total }=\mathrm{p}, \\
\mathrm{a,b} \text { Valores ce } \\
\text { x,y Valores c }\end{array}$ & 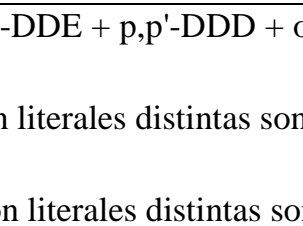 & $\begin{array}{c}\text { p'-DDT + p,p'-DDT; } \\
\text { HCH. } \\
\text { diferentes significativ } \\
\text { tratamiento. }\end{array}$ & $\mathrm{ICH}$ total $=\alpha-\mathrm{HCH}$ & $\begin{array}{l}\text { 3-HCH }+\gamma-\mathrm{HCH}+\delta \\
\text { olumnas del mismo }\end{array}$ \\
\hline
\end{tabular}

Como se aprecia en el Cuadro 2, estas concentraciones detectadas en la leche bronca fueron menores a las registradas en leche bronca de bovino en el estudio anterior realizado por Pardío et $a l^{(40)}$ en el estado de Veracruz. Comparando nuestros datos con las concentraciones 
de los metabolitos del DDT detectados en leche bronca de Brasil $^{(24)}$, Colombia ${ }^{(14)}$ y Rumania ${ }^{(27)}$, se observa que los niveles detectados en las leches control de $63{ }^{\circ} \mathrm{C}$ y $73{ }^{\circ} \mathrm{C}$ en el presente estudio fueron hasta 26 y 11 veces más altos, respectivamente. Sin embargo, en Egipto la concentración de p,p'-DDT en leche bronca fue dos veces mayor que la encontrada en los testigos en esta investigación. Con respecto a las concentraciones de $\beta-\mathrm{HCH}$ y $\gamma-\mathrm{HCH}$ $\left(1.438 \pm 0.376\right.$ y $1.187 \pm 0.204 \mu \mathrm{g} \mathrm{kg}^{-1}$ base lipídica, respectivamente) de la leche control de $73{ }^{\circ} \mathrm{C}$ fueron $(P<0.05)$ más altas con respecto a las concentraciones del control de $63{ }^{\circ} \mathrm{C}$ $\left(0.401 \pm 0.276\right.$ y $0.511 \pm 0.338 \mu \mathrm{g} \mathrm{kg}^{-1}$ base lipídica, respectivamente). No obstante que el $\mathrm{HCH}$ total de la leche control de $73{ }^{\circ} \mathrm{C}$ fue 2.24 veces mayor que el de $63{ }^{\circ} \mathrm{C}$, no fue significativamente $(P>0.05)$. La variación de las concentraciones de los POC entre los controles pudo haberse debido a que sus niveles en la leche de cada UP pudieron haber variado indistintamente a lo largo del ciclo anual. Esta variación podría atribuirse a la combinación de las prácticas de manejo zootécnico en las unidades de producción y a las dietas formuladas con ingredientes contaminados, el efecto de la cinética debido a las propiedades fisicoquímicas de cada isómero, las actividades metabólicas relacionadas con la movilización lipídica, y posiblemente la falta de homogeneidad en la concentración de los metabolitos debido a la remobilización de los lípidos y contaminantes durante la síntesis de la leche, así como a la carga de aplicación del plaguicida ya que éste puede ser depositado o absorbido desde la atmosfera hacia la superficie del pasto, siendo afectado por la temperatura, la lluvia, el viento y el clima ${ }^{(2)}$.

Los metabolitos $\alpha-\mathrm{HCH}, \beta-\mathrm{HCH}, \gamma-\mathrm{HCH}$ y $\mathrm{HCH}$ total presentes en los controles de $63{ }^{\circ} \mathrm{C}$ y $73{ }^{\circ} \mathrm{C}$ no rebasaron los LMR de 50, 20, 10 y $100 \mu \mathrm{g} \mathrm{kg}^{-1}$ (base lipídica), respectivamente, establecidos por la $\mathrm{FAO} / \mathrm{WHO}^{(39)}$. Como se aprecia en el Cuadro 2, las concentraciones de los metabolitos $\alpha-\mathrm{HCH}, \beta-\mathrm{HCH}$ y $\gamma-\mathrm{HCH}$ evaluados en el presente estudio fueron menores a las reportadas por Pardío et al ${ }^{(40)}$ en Veracruz, México; los niveles de $\gamma$-HCH reportados en leche de Tlalixcoyan, Ver., fueron 250.3 veces mayor a lo reportado en la leche control de $63{ }^{\circ} \mathrm{C}\left(0.511 \pm 0.338 \mu \mathrm{g} \mathrm{kg}^{-1}\right.$ base lipídica) y 107.76 veces mayor a la leche control de $73{ }^{\circ} \mathrm{C}$ $\left(1.187 \pm 0.204 \mu \mathrm{g} \mathrm{kg}^{-1}\right.$ base lipídica). La concentración de $\gamma$-HCH en Egipto fue 62.5 veces mayor a la reportada en la leche bronca a $63{ }^{\circ} \mathrm{C}$ y 26.9 veces mayor en la leche control de $73{ }^{\circ} \mathrm{C}$. Sin embargo, la concentración de $\alpha-\mathrm{HCH}$ registrada en la leche de Colombia fue similar a la observada en la leche control a $73^{\circ} \mathrm{C}\left(0.310 \pm 0.111 \mu \mathrm{g} \mathrm{kg}{ }^{-1}\right.$ base lipídica $)$. 
Cuadro 2: Residuos de plaguicidas organoclorados $\left(\mu \mathrm{g} \mathrm{kg}^{-1}\right.$ base lipídica) en leche bronca reportados en otros estudios

\begin{tabular}{|c|c|c|c|c|c|c|c|c|c|}
\hline Localidad/País & $\begin{array}{l}\text { p,p'- } \\
\text { DDE }\end{array}$ & $\begin{array}{l}\text { p,p'- } \\
\text { DDD }\end{array}$ & $\begin{array}{l}\text { p,p'- } \\
\text { DDT }\end{array}$ & $\begin{array}{l}\text { o,p'- } \\
\text { DDT }\end{array}$ & $\begin{array}{l}\alpha- \\
\mathrm{HCH}\end{array}$ & $\begin{array}{l}\beta- \\
\mathrm{HCH}\end{array}$ & $\begin{array}{l}\gamma- \\
\mathbf{H C H}\end{array}$ & $\begin{array}{l}\delta- \\
\text { HCH }\end{array}$ & cia \\
\hline $\begin{array}{l}\text { Medellín, } \\
\text { Veracruz/México }\end{array}$ & 39.000 & ND & 89.000 & 26.000 & 13.000 & 23.000 & 49.000 & NA & $\begin{array}{l}\text { Pardío } \\
a l^{(40)}\end{array}$ \\
\hline $\begin{array}{l}\text { Paso San Juan, } \\
\text { Veracruz/México }\end{array}$ & 0 & ND & 0 & ND & 00 & 17.000 & 22.000 & NA & $\begin{array}{l}\text { Pardío } \\
a l^{(40)}\end{array}$ \\
\hline $\begin{array}{l}\text { Tlalixcoyan, } \\
\text { Veracruz/México }\end{array}$ & 24.000 & ND & 36.000 & ND & 31.00 & 69.000 & 128.000 & NA & $\begin{array}{l}\text { Pardío } \\
a l^{(40)}\end{array}$ \\
\hline $\begin{array}{l}\text { Rio Grande do } \\
\text { Sul/Brasil }\end{array}$ & 0.011 & 0.000 & ND & 0.000 & 0.002 & ND & 0.006 & ND & $\begin{array}{l}\text { Heck } \\
a l^{(24)}\end{array}$ \\
\hline Cair & 12.000 & 6.000 & 24.000 & 3.000 & $\mathrm{NA}$ & NA & 32.000 & $\mathrm{NA}$ & $\begin{array}{l}\text { Abou-Arab } \\
\text { et } a l^{(26)}\end{array}$ \\
\hline $\begin{array}{l}\text { Sabanas, } \\
\text { Córdoba/Colombia }\end{array}$ & ND & ND & 0.034 & ND & 0.469 & ND & ND & ND & $\begin{array}{l}\text { Díaz } \\
a l^{(14)}\end{array}$ \\
\hline Rumania & 0.011 & 0.000 & 0.000 & 0.000 & 0.001 & 0.010 & 0.002 & 0.001 & $\begin{array}{l}\text { Miclean et } \\
a l^{(27)}\end{array}$ \\
\hline
\end{tabular}

$\mathrm{NA}=$ no analizado $\mathrm{ND}=$ no detectado.

Cabe destacar que los plaguicidas organoclorados y sus residuos son de naturaleza altamente lipofílica y persistente, por lo que se concentran fácilmente en la grasa de la leche ${ }^{(26)}$. Esto implica que, aunque los POC se prohibieron para el uso agrícola a principios de la década de 1970 en la mayoría de los países, sus residuos aún persisten. Debido a su persistencia, las concentraciones detectadas de DDT, HCH y sus metabolitos en la leche bronca del presente estudio son debidas probablemente a las cargas aplicadas de DDT durante su uso legal anterior, mismo que ha provocado la contaminación de las áreas de pastoreo de bovinos en el estado de Veracruz. Así mismo, el $\gamma-\mathrm{HCH}$ continúa siendo utilizado para el control de ectoparásitos en el ganado ${ }^{(2)}$. De hecho, estos plaguicidas se siguen aplicando en varias partes del mundo debido a sus efectos potentes y de amplio espectro contra organismos nocivos ${ }^{(41)}$.

\section{Efecto de la pasteurización en los niveles de concentración de DDT y $\mathrm{HCH}$}

Como se aprecia en los Cuadros 1 y 3, después de la pasteurización a $63^{\circ} \mathrm{C}$, los niveles de concentración de los metabolitos p,p'-DDE y o,p'-DDT (10.673 \pm 5.682 y $7.559 \pm 4.015 \mu \mathrm{g}$ $\mathrm{kg}^{-1}$ base lipídica, respectivamente) disminuyeron $(P<0.05)$ con respecto a los niveles de la leche control (bronca) un 32.17 y $50.39 \%$, respectivamente, y sólo el p,p'DDT incrementó $(P>0.05) 0.31 \%$ su concentración con respecto al control; por lo que la concentración del DDT total $\left(20.170 \pm 16.050 \mu \mathrm{g} \mathrm{kg}^{-1}\right.$ base lipídica) disminuyó ( $\left.P>0.05\right) 29.83 \%$ con respecto a la del control $\left(26.480 \pm 13.880 \mu \mathrm{g} \mathrm{kg}^{-1}\right.$ base lipídica $)$. Sin embargo, los niveles de $\alpha-\mathrm{HCH}$ 
y $\beta$-HCH $\left(0.355 \pm 0.137\right.$ y $0.919 \pm 0.062 \mu \mathrm{g} \mathrm{kg}^{-1}$ base lipídica, respectivamente $)$ se incrementaron $(P<0.05) 31.48$ y $129.11 \%$, respectivamente, pero el incremento de los niveles de $\gamma-\mathrm{HCH}, \delta-\mathrm{HCH}$ y $\mathrm{HCH}$ total no fue significativo $(P>0.05)$. Los isómeros $\alpha-\mathrm{HCH}$ y $\gamma-\mathrm{HCH}$ se pueden isomerizar a $\beta-\mathrm{HCH}$. La estabilidad del $\beta-\mathrm{HCH}$, su tendencia a acumularse en los tejidos humano y animal con el tiempo, su rápida bio-concentración en el hombre (525 veces) y su eliminación más lenta, representa un riesgo respecto a su toxicidad crónica. Este metabolito es el más tóxico seguido por el $\alpha-, \gamma-\mathrm{HCH}$ y $\delta-\mathrm{HCH}$ debido a su mayor vida media biológica en el cuerpo de 7-8 años (ATSDR) ${ }^{(42)}$.

Cuadro 3: Variación (\%) de la concentración de DDT, HCH y sus metabolitos debido al efecto de pasteurización de la leche de ganado bovino en diversos estudios

\begin{tabular}{|c|c|c|c|c|c|c|}
\hline \multirow{2}{*}{$\begin{array}{l}\text { Tipo de } \\
\text { muestra/Procedencia }\end{array}$} & \multirow[t]{2}{*}{ PLAG } & \multicolumn{2}{|c|}{ 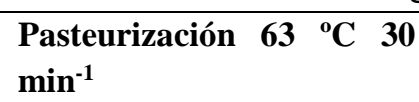 } & \multicolumn{2}{|c|}{$\begin{array}{lllll}\begin{array}{l}\text { Pasteurización } \\
\text { seg }^{-1}\end{array} & 73 & & & \\
\end{array}$} & \multirow[t]{2}{*}{ Referencia } \\
\hline & & Disminución & Incremento & Disminución & Incremento & \\
\hline \multirow[t]{3}{*}{$\begin{array}{l}\text { Leche } \\
\text { de búfalo/Egipto }\end{array}$} & $\begin{array}{l}\text { p,p'- } \\
\text { DDE }\end{array}$ & NA & NA & 23.07 & $\begin{array}{ll}-- \\
\end{array}$ & \multirow[t]{3}{*}{$\begin{array}{l}\text { Abd-Rabo et } \\
a l^{(21)}\end{array}$} \\
\hline & $\begin{array}{l}\mathrm{p}, \mathrm{p}^{\prime}- \\
\text { DDD }\end{array}$ & NA & NA & --- & 30 & \\
\hline & $\begin{array}{l}\mathrm{p}, \mathrm{p}^{\prime}- \\
\text { DDT }\end{array}$ & NA & NA & 32.85 & --- & \\
\hline \multirow[t]{2}{*}{ Leche bovina/Italia } & $\begin{array}{l}\text { DDT } \\
\text { total }^{\circ}\end{array}$ & NA & NA & --- & 6.5 & \multirow[t]{2}{*}{$\begin{array}{l}\text { Deiana y } \\
\text { Fatichenti }^{(22)}\end{array}$} \\
\hline & $\begin{array}{l}\mathrm{HCH} \\
\text { total }^{\square}\end{array}$ & NA & $\mathrm{NA}$ & --- & 6.5 & \\
\hline Leche bovina/Egipto & $\gamma-\mathrm{HCH}$ & 72.90 & --- & 65.00 & --- & $\begin{array}{l}\text { Abou-Arab } \\
\text { (23) }\end{array}$ \\
\hline \multirow[t]{10}{*}{$\begin{array}{l}\text { Leche } \\
\text { bovina/México }\end{array}$} & $\begin{array}{l}\mathrm{p}, \mathrm{p}^{\prime}- \\
\mathrm{DDE}\end{array}$ & 32.17 & --- & 30.94 & --- & \multirow[t]{10}{*}{$\begin{array}{l}\text { Presente } \\
\text { estudio }\end{array}$} \\
\hline & $\begin{array}{l}\mathrm{p}, \mathrm{p}^{\prime}- \\
\text { DDD }\end{array}$ & 15.38 & --- & 44.51 & --- & \\
\hline & $\begin{array}{l}\text { o,p'- } \\
\text { DDT }\end{array}$ & 50.39 & --- & 3.18 & --- & \\
\hline & $\begin{array}{l}\mathrm{p}, \mathrm{p}^{\prime}- \\
\text { DDT }\end{array}$ & --- & 0.31 & 81.23 & --- & \\
\hline & $\begin{array}{l}\text { DDT } \\
\text { total }^{\circ}\end{array}$ & 29.83 & & 42.82 & --- & \\
\hline & $\alpha-\mathrm{HCH}$ & --- & 31.48 & --- & 75.16 & \\
\hline & $\beta-\mathrm{HCH}$ & --- & 129.11 & 85.68 & --- & \\
\hline & $\gamma-\mathrm{HCH}$ & --- & 37.37 & 18.88 & --- & \\
\hline & $\delta$ - $\mathrm{HCH}$ & --- & 257.24 & --- & 187.57 & \\
\hline & $\begin{array}{l}\mathrm{HCH} \\
\text { total }^{\square}\end{array}$ & --- & 93.29 & 99.31 & --- & \\
\hline
\end{tabular}

PLAG $=$ plaguicida; $\mathrm{NA}=$ no analizado $;{ }^{\circ} \mathrm{DDT}$ total $=\mathrm{p}, \mathrm{p}-\mathrm{DDE}+\mathrm{p}, \mathrm{p}^{\prime}-\mathrm{DDD}+\mathrm{o}, \mathrm{p}^{\prime}-\mathrm{DDT}+\mathrm{p}, \mathrm{p}^{\prime}-\mathrm{DDT} ;{ }^{\square} \mathrm{HCH}$ total $=\alpha-\mathrm{HCH}+\beta-\mathrm{HCH}+\gamma-\mathrm{HCH}+\delta-\mathrm{HCH}$. 
La pasteurización a $73{ }^{\circ} \mathrm{C}$ disminuyó $(P<0.05)$ los niveles de concentración de DDT y sus metabolitos en la leche con respecto a la leche testigo, disminuyendo desde 3.18 \% (o,p'DDT) hasta un $81.23 \%$ (p,p'-DDT). Se observó un incremento significativo $(P<0.05)$ en los niveles de $\alpha-\mathrm{HCH}$ y d-HCH $\left(0.543 \pm 0.151\right.$ y $1.504 \pm 2.379 \mu \mathrm{g} \mathrm{kg}^{-1}$ base lipídica, respectivamente) en 75.16 y $185.57 \%$, respectivamente, y una disminución significativa $(P<0.05)$ para $\beta$-HCH $\left(0.206 \pm 0.113 \mu \mathrm{g} \mathrm{kg}^{-1}\right.$ base lipídica $)$ en $85.68 \%$. La disminución observada en las concentraciones de p,p'-DDE, p,p'-DDD, o,p'-DDT y DDT total en la leche pasteurizada a $63{ }^{\circ} \mathrm{C}$ en comparación con la concentración del control, excepto el p,p'-DDT pudiera atribuirse a la isomerización del p,p'-DDE y del p,p'-DDD a p,p'-DDT por el calentamiento. Sin embargo, las concentraciones de $\mathrm{HCH}$ y todos sus metabolitos se incrementaron con la pasteurización a $63{ }^{\circ} \mathrm{C}$ y $\operatorname{los}$ metabolitos $\alpha-\mathrm{HCH}$ y $\delta$-HCH por la pasteurización a $73{ }^{\circ} \mathrm{C}$. Los plaguicidas organoclorados pueden ser degradados por fotólisis, hidrólisis, oxidación y reducción, temperatura y $\mathrm{pH}$. La retención de los plaguicidas dependerá de las propiedades físico-químicas de la molécula del plaguicida así como del alimento $^{(43)}$. No obstante, la concentración del DDT total de la leche control y pasteurizada a 63 y $73{ }^{\circ} \mathrm{C}$ no rebasó el LMR de $50 \mu \mathrm{g} \mathrm{kg}^{-1}$ (base lipídica) establecido por la $\mathrm{FAO} / \mathrm{WHO}^{(39)}$. Así mismo, los metabolitos $\alpha-\mathrm{HCH}, \beta-\mathrm{HCH}, \gamma-\mathrm{HCH}$ y $\mathrm{HCH}$ total presentes en las leches pasteurizadas a $63{ }^{\circ} \mathrm{C}$ y $73{ }^{\circ} \mathrm{C}$ no rebasaron los LMR de 50, 20, 10 y $100 \mu \mathrm{g} \mathrm{kg}^{-1}$ (base lipídica), respectivamente, establecidos por la FAO/ $\mathrm{WHO}^{(39)}$. Sin embargo, el metabolito $\delta$ $\mathrm{HCH}$ cuyos niveles se incrementaron en ambas pasteurizaciones está estructuralmente relacionado a los $\mathrm{HCH}$ cancerígenos $(\mathrm{ATSDR})^{(42)}$.

Los resultados de la pasteurización a 63 y $73{ }^{\circ} \mathrm{C}$ difieren con los de Abou-Arab ${ }^{(23)}$ quien reportó una disminución de 72.9 y $65.0 \%$, respectivamente, en la concentración de $\gamma$-HCH en leche de bovino, mientras que en nuestro estudio este metabolito incrementó $37.37 \%$ con la pasteurización a $63{ }^{\circ} \mathrm{C}$ pero disminuyó $18.88 \%$ con la pasteurización a $73{ }^{\circ} \mathrm{C}$. Abd-Rabo et $a l^{(21)}$ en Egipto reportó que los metabolitos p,p'-DDE y p,p'-DDT disminuyeron en la leche pasteurizada a $73{ }^{\circ} \mathrm{C}$. En el presente estudio se observó un disminución similar en el p,p'DDE pero un incremento en el p,p'-DDT. La disminución observada en el p,p'-DDD $(44.51 \%)$ en este estudio contrasta con el incremento reportado por este autor (30\%). Deiana y Fatichenti $^{(22)}$ en Italia reportaron que las concentraciones del DDT y $\mathrm{HCH}$ totales incrementaron el $6.50 \%$ en leche bovina pasteurizada a $73{ }^{\circ} \mathrm{C}$, mientras que en el presente estudio disminuyeron 42.82 y $99.31 \%$, respectivamente. Estos resultados indican que la pasteurización a $73{ }^{\circ} \mathrm{C} 15 \mathrm{seg}^{-1}$ disminuye la concentración de la mayoría de los POC analizados. Es importante señalar que la leche está considerada como un producto de primera necesidad en la alimentación humana. Los beneficios de la leche bovina no se limitan a su valor nutricional, constituyen un factor de prevención de patologías como la enfermedad cardiovascular, algunos tipos de cáncer, la hipertensión arterial y ósea o dental ${ }^{(20)}$. De ahí la importancia de la disminución de la concentración de estos contaminantes en la leche por la pasteurización, ya que su presencia representa un riesgo a la salud pública. 


\section{Estimación de la exposición dietaria de DDT y HCH a través del consumo humano de leche de bovino}

\section{Ingesta diaria estimada (IDE) para DDT y $\mathrm{HCH}$}

Con la finalidad de proteger la salud pública se han establecido ingestas límites de POC en leche y otros alimentos que no deben superarse. La ingesta diaria aceptable (IDA) para la DDT total es de $20 \mu \mathrm{g} \mathrm{kg}^{-1}$ pc día ${ }^{-1}$ recomendado por la $\mathrm{FAO} / \mathrm{WHO}^{(39)}$ y $0.5 \mu \mathrm{g} \mathrm{kg}^{-1}$ pc día ${ }^{-1}$ recomendado por la $\mathrm{EPA}^{(44)}$, para $\gamma-\mathrm{HCH}$ (lindano) es de $8 \mu \mathrm{g} \mathrm{kg}^{-1} \mathrm{pc} \mathrm{día}^{-1(39)}$. Por lo tanto, la IDE se calculó para el DDT total y el metabolito $\gamma$-HCH a partir de la concentración detectada en las leches estudiadas para tres grupos de población: niños, adultos y ancianos.

En el Cuadro 4 se observa que en el grupo de los niños la mayor IDE se calculó en la leche control de $63{ }^{\circ} \mathrm{C}$, que no rebasó los valores aceptables recomendados por la FAO/WHO (20 $\mu \mathrm{g} \mathrm{kg}^{-1}$ pc día ${ }^{-1}$ ), pero fue 28.06 veces más alta que los niveles recomendados por la EPA $\left(0.5 \mu \mathrm{g} \mathrm{kg}^{-1} \mathrm{pc} \mathrm{día}{ }^{-1}\right)$. En el grupo de los adultos la mayor concentración también ocurrió en la leche control de $63{ }^{\circ} \mathrm{C}$, sin rebasar los valores recomendados por la FAO/WHO; sin embargo, rebasó 10 veces los valores aceptables de la EPA. La IDE del grupo de ancianos fue mayor para la leche control de $63{ }^{\circ} \mathrm{C}$, rebasando 13.74 veces los valores aceptables por la EPA. Abou-Arab et al ${ }^{(26)}$ estimaron IDE de DDT total de 0.394 y $0.113 \mu \mathrm{g} \mathrm{kg}^{-1} \mathrm{pc} \mathrm{día}{ }^{-1}$ para adultos y de 0.475 y $0.135 \mu \mathrm{g} \mathrm{kg}^{-1} \mathrm{pc}$ día $^{-1}$ para niños, así mismo las IDEs de $\gamma-\mathrm{HCH}$ fueron 0.280 y $0.113 \mu \mathrm{g} \mathrm{kg}^{-1}$ pc día ${ }^{-1}$ para adultos y 0.336 y $0.135 \mu \mathrm{g} \mathrm{kg}^{-1}$ pc día ${ }^{-1}$ para niños por el consumo de leche bronca y pasteurizada $\left(63^{\circ} \mathrm{C}\right)$, respectivamente, procedente de mercados locales en el Cairo, Egipto. De acuerdo con esto, las IDE del presente estudio fueron menores a las estimadas en adultos y niños que consumen leche bronca y pasteurizada en Egipto, debido a que las concentraciones de DDT y $\mathrm{HCH}$ en esa zona fueron más altas que las reportadas en la presente investigación. 
Cuadro 4: Ingesta diaria estimada (IDE) $\left(\mu \mathrm{g} \mathrm{kg}^{-1} \mathrm{pc} \mathrm{día}{ }^{-1}\right)$ de DDT total y $\gamma$-HCH para niños, adultos y ancianos estimada en el consumo de leche bronca (control) y pasteurizada

\begin{tabular}{|c|c|c|c|c|c|}
\hline \multirow{2}{*}{$\begin{array}{l}\text { Grupo de } \\
\text { población }\end{array}$} & \multicolumn{2}{|c|}{ Tratamiento $63^{\circ} \mathrm{C} 30 \mathrm{~min}^{-1}$} & \multicolumn{2}{|c|}{ Tratamiento $73{ }^{\circ} \mathrm{C} 15 \mathrm{sec}^{-1}$} & \multirow{2}{*}{ 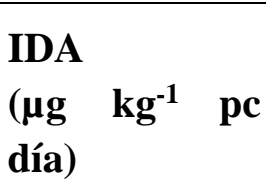 } \\
\hline & $\begin{array}{l}\text { Leche } \\
\text { control }\end{array}$ & $\begin{array}{l}\text { Leche } \\
\text { pasteurizada }\end{array}$ & $\begin{array}{l}\text { Leche } \\
\text { control }\end{array}$ & $\begin{array}{l}\text { Leche } \\
\text { pasteurizada }\end{array}$ & \\
\hline DDT total & & & & & $20^{(39)}$ y $0.05^{(44)}$ \\
\hline Niños & 14.03 & 10.68 & 4.72 & 2.70 & \\
\hline Adultos & 5.00 & 3.81 & 1.62 & 0.96 & \\
\hline Ancianos & 6.87 & 5.23 & 2.31 & 1.32 & \\
\hline$\gamma-\mathrm{HCH}$ & & & & & $8^{(39)}$ \\
\hline Niños & 0.27 & 0.37 & 0.62 & 0.50 & \\
\hline Adultos & 0.04 & 0.06 & 7.81 & 0.09 & \\
\hline Ancianos & 0.08 & 0.11 & 0.19 & 0.16 & \\
\hline
\end{tabular}
$\mathrm{IDA}=$ ingesta diaria aceptable; FAO/WHO, $1997^{(39)} ; \mathrm{EPA}^{(44)}$. DDT total $=p, p^{\prime}-D D E+p, p^{\prime}-D D D+o, p^{\prime}-D D T+p, p^{\prime}-D D T$.

La IDE más alta $\gamma-\mathrm{HCH}$ en los tres grupos de población se presentó en la leche control de $73{ }^{\circ} \mathrm{C}$. Sin embargo, ninguna de las IDEs rebasó el valor aceptable recomendado por la FAO/WHO ( $8 \mu \mathrm{g} \mathrm{kg}^{-1}$ pc por día). Pardío et $a l^{(40)}$ reportaron que las IDE para infantes y adultos por el consumo de leche cruda de Tlalixcoyan, Veracruz, México, contaminada con $\gamma$-HCH fue de 0.666 y $0.021 \mu \mathrm{g} \mathrm{kg}^{-1} \mathrm{pc} \mathrm{día}^{-1}$, respectivamente. Sin embargo, las IDE para infantes y adultos por el consumo de leche cruda contaminada con DDT total fueron más altas para la leche procedente de Medellín, Veracruz, México con valores de 0.530 y 0.017 $\mu \mathrm{g} \mathrm{kg}{ }^{-1}$ pc día ${ }^{-1}$, respectivamente. En un estudio reciente, Miclean et al ${ }^{(27)}$ reportó IDEs de $\mathrm{HCH}$ total en leche bronca para mujeres, hombres y niños de $0.002,0.002$ y $0.012 \mu \mathrm{g} \mathrm{kg}^{-1} \mathrm{pc}$ día $^{-1}$, respectivamente; para DDT total fueron 0.001, 0.002 y $0.008 \mu \mathrm{g} \mathrm{kg}^{-1}$ pc día ${ }^{-1}$ para mujeres, hombres y niños, respectivamente, menores a las estimadas para el DDT total en niños en el presente estudio por el consumo de leche pasteurizada a 63 y $73{ }^{\circ} \mathrm{C}$ y bronca (controles respectivos). Al comparar los resultados de Pardío et al ${ }^{(40)}$ con los del presente estudio, se observa que la exposición a $\gamma-\mathrm{HCH}$ y a DDT total a través del consumo de leche bronca ha aumentado en la zona. Lo anterior indica un incremento de la contaminación en el tiempo debido al uso continuo del $\gamma-\mathrm{HCH}$ en la ganadería en la región y a las altas cargas de DDT rociadas en el pasado que resultaron en pastizales contaminados cercanos a las zonas urbanas y suburbanas, donde el DDT era rociado para el control del paludismo ${ }^{(2)}$. Sin embargo, el 30 a $37 \%$ de la leche fluida sin pasteurizar de la producción nacional se destina a la producción de quesos artesanales ${ }^{(45)}$, mientras que, de la producción en el estado de Veracruz, el $50 \%$ de la leche fluida no pasteurizada en acopios se venden a queserías locales para la producción de quesos y otros productos lácteos artesanales que se comercializan en 
las principales zonas urbanas de la entidad ${ }^{(46)}$. Como consecuencia, los consumidores de la leche producida en esta zona agraria de Veracruz están expuestos a niveles dietarios de POs superiores a los niveles de exposición en países desarrollados, en donde el uso de estos plaguicidas fue prohibido desde hace muchos años ${ }^{(40)}$. Este riesgo podría reducir si la leche fuera pasteurizada a $73{ }^{\circ} \mathrm{C} 15 \mathrm{seg}^{-1}$ para disminuir la concentración de la mayoría de los POC analizados. Como se aprecia en el cuadro 3, de acuerdo con la literatura consultada, son muy escasos los estudios realizados en pasteurización lenta y rápida y la estimación de la ingesta dietaria respectiva. La vigilancia de los niveles de estos POC en la leche y en otros alimentos resulta indispensable para observar que no se sobrepasen los LMR y las IDA recomendadas por la FAO/WHO.

\section{Dosis diaria promedio (DDP) estimada para DDT}

La DDP es una predicción de la ingesta diaria de residuos de un plaguicida basada en la estimación de las concentraciones de residuos en los alimentos y en los datos disponibles sobre el consumo de los alimentos en relación con una población determinada ${ }^{(47)}$. Para el DDT total, la Organización de las Naciones Unidas para la Agricultura y la Alimentación (FAO) y la Organización Mundial de la Salud (OMS) han establecido la DDP, de $48 \mu \mathrm{g} \mathrm{kg}^{-1}$ día $^{-1(45)}$. En el caso de HCH no hay una DDP establecida.

Las DDP más altas estimadas en niños, adultos y ancianos fueron de 142.01, 71.00 y 91.72 $\mu \mathrm{g} \mathrm{kg}{ }^{-1}$ día $^{-1}$, respectivamente, debido a la ingesta de leche control (bronca) de $63{ }^{\circ} \mathrm{C}$, sobrepasando el límite recomendado por la FAO/WHO $\left(48 \mu \mathrm{g} \mathrm{kg}^{-1} \mathrm{día}^{-1}\right)$ por 2.9 veces el grupo de los niños, 1.4 veces los adultos y 1.9 veces los ancianos. Después de pasteurizar esta leche a $63{ }^{\circ} \mathrm{C}$, las DDP calculadas en los tres grupos de población (114.06, 57.03 y 73.66 $\mu \mathrm{g} \mathrm{kg}^{-1}$ día $^{-1}$ en niños, adultos y ancianos, respectivamente) también rebasaron el límite recomendado. Cabe destacar que la DDP estimada por consumo de leche control de $73^{\circ} \mathrm{C}$ en niños fue de $59.48 \mu \mathrm{g} \mathrm{kg}^{-1}$ día $^{-1}$, mayor al límite recomendado por la FAO/WHO $(48 \mu \mathrm{g})$; sin embargo, una vez pasteurizada la leche el valor de DDP resultó 6.15 veces menor $(9.67 \mu \mathrm{g})$ en comparación a la leche testigo. Las DDP calculadas para los adultos y ancianos en la leche control (29.74 y $38.41 \mu \mathrm{g} \mathrm{kg}^{-1}$ día $^{-1}$, respectivamente) y pasteurizada a $73{ }^{\circ} \mathrm{C}(18.08$ y 23.35 $\mu \mathrm{g}$, respectivamente) permanecieron por debajo del límite recomendado por la FAO/WHO y fueron menores a las estimadas por el consumo de leche bronca utilizada como control a $63{ }^{\circ} \mathrm{C}$. Estos resultados contrastan con los reportados por Pardío et a $l^{(40)}$ quienes estimaron menores dosis diarias para infantes y adultos (4.068 y $2.339 \mu \mathrm{g} \mathrm{kg}^{-1} \mathrm{día}^{-1}$, respectivamente) que consumían leche bronca de Medellín, Veracruz, México. Se debe destacar que existen determinados grupos de población más vulnerables a los efectos de estos plaguicidas, como son la población infantil, primordialmente aquéllos que poseen algún grado de desnutrición y la población femenina en edad fértil, particularmente en estado de gestación, ya que existe evidencia de su actividad de disrupción hormonal y lipídica ${ }^{(48)}$. Los efectos de la exposición 
a POC a la salud humana a través de los alimentos es un problema que merece más atención. Los resultados obtenidos indican su presencia en la leche bronca y pasteurizada y un incremento en las DDP, por lo que resulta indispensable la revisión de los LMR y la búsqueda de métodos alternativos de control de plagas para mejorar la inocuidad alimentaria y proteger la salud pública.

\section{Conclusiones e implicaciones}

El proceso de pasteurización a $73{ }^{\circ} \mathrm{C}$ de leche disminuye las concentraciónes de DDT y sus metabolitos, así como la mayoría de las concentraciones de los metabolitos de HCH. Por lo tanto, el proceso térmico bajo estas condiciones representa una alternativa favorable para la dismunución de la exposición dietaria a estos plaguicidas por consumo humano de leche pasteurizada a $73{ }^{\circ} \mathrm{C}$.

\section{Agradecimientos}

Se agradece al proyecto "Formación y Fortalecimiento de Cuerpos Académicos e Integración de Redes" clave 103.5/03/477 UVER-F-11 fondos PRODEP por el financiamiento de este estudio.

\section{Literatura citada:}

1. Negi RK, Rani S. Contamination profile of DDT and HCH in packaged milk samples collected from Haridwar, India. Int J Pure App Biosci 2015;3:121-127.

2. Pardío V, Martínez D, Flores A, Romero D, Suárez V, López K, et al. Human health risk of dietary intake of organochlorine pesticide residues in bovine meat and tissues from Veracruz, México. Food Chem 2012;135:1873-1893.

3. Avalos GM, Ramírez GJ. La situación del lindano en México. Gaceta Ecol 2003;69:93100. http://www.redalyc.org/pdf/539/53906907.pdf. Consultado 29 Abr, 2019.

4. Vijgen J, Abhilash PC, Fan LY, Lal R, Forter M, Torres J, et al. Hexachlorocyclohexane $(\mathrm{HCH})$ as new Stockholm Convention POPs a global perspective on the management of Lindane and its waste isomers. Environ Sci Pollut Res 2011;18:152-162.

5. Mendoza CA, Ize LIAR. Las sustancias químicas en México. Perspectivas para un manejo adecuado. Rev Int Contam Ambie 2017;33:719-745.

6. Ocampo-Camberos L, Rosiles-Martínez R, Tapia-Pérez G, Sumano-López H. Cinética de eliminación de Lindano en grasa de leche de vacas tratadas con tres dosis de Lindano. Agrociencia 2010;44:461-469. 
7. Kalantzi OI, Alcock RE, Johnston PA, Santillo D, Stringer RL, Thomas GO, et al. The global distribution of PCBs and organochlorine pesticides in butter. Environ Sci Technol 2001;35:1013-1018.

8. Vieira DRE, Torres JPM, Malm O. DDT environmental persistence from its use in a vector control program: a case study. Environ Res Section A 2001;86:174-182.

9. Nag SK, Raikwar MK. Organochlorine pesticide residues in bovine milk. Bull Environ Contam Toxicol 2008;80:5-9.

10. Tsakiris IN, Goumenou M, Tzatzarakis MN, Alegakis AK, Tsitsimpikou C, Ozcagli E, et al. Risk assessment for children exposed to DDT residues in various milk types from the Greek market. Food Chem Toxicol 2015;75:156-165.

11. Yentür G, Kalay A, Öktem AB. A survey on organochlorine pesticide residues in butter and cracked wheat available in Turkish markets. Nahrung 2001;45:40-42.

12. Yadav ICh, Devi NL, Syed JH, Cheng Z, Li J, Zhang G, et al. Current status of persistent organic pesticides residues in air, water, and soil, and their possible effect on neighboring countries: A comprehensive review of India. Sci Total Environ 2015;511:123-137.

13. Jandacek RJ, Tso P. Factors affecting the storage and excretion of toxic lipophilic xenobiotics. Lipids 2001;36:1289-1305.

14. Díaz-Pongutá B, Lans-Ceballos E, Barrera-Violeth JL. Organochlorine insecticide residues present in raw milk sold in the Department Córdoba, Colombia. Acta Agron 2012;61(1):10-15.

15. Kuba J, Tomza-Marciniak A, Pilarczyk B, Tarasewicz N, Pilarczyk, Ligocki M. Comparison of DDT and its metabolites concentrations in cow milk from agricultural and industrial areas. J Environ Sci Health B 2015;50:1-7.

16. Battu RS, Balwinder S, Kang BK, Joia BS. Risk assessment through dietary intake of total diet contaminated with pesticide residues in Punjab, India, 1999-2002. Ecotoxicol Environ Saf 2005;62:132-139.

17. Aamir M, Khan S, Li G. Dietary exposure to $\mathrm{HCH}$ and DDT congeners and their associated cancer risk based on Pakistani food consumption. Environ Sci Pollut Res 2018;25(9):8465-8474.

18. SAGARPA/SIAP. Secretaría de Agricultura, Ganadería, Desarrollo Rural, Pesca y Alimentación/Servicio de Información Agroalimentaria y Pesquera. Panorama de la leche en México. México. 2018. 
19. USDA/WASDE. United States Department of Agriculture/World Agricultural Supply and Demand Estimates. Dairy: world market and trade. United States. 2018.

20. Fernández FE, Martínez HJA, Martínez SV, Moreno VJM, Collado YLR, Hernández CM, et al. Documento de Consenso: importancia nutricional y metabólica de la leche. Nutr Hosp 2015;31:92-101.

21. Abd-Rabo FH, Abou DAE, Ahmed NS, Hassan FAM. Heat treatments change amounts of pesticide residues in buffaloe's milk. Egypt J Dairy Sci 1989;17:349-357.

22. Deiana P, Fatichenti F. Pesticide residues in milk processing. Ital J Food Sci 1992;4:229245.

23. Abou-Arab AAK. Effects of processing and storage of dairy products on lindane residues and metabolites. Food Chem 1999;64:467-473.

24. Heck MC, Sifuentes dos Santos J, Bogusz Junior S, Costabeber I, Emanuelli T. Estimation of children exposure to organochlorine compounds through milk in Rio Grande do Sul, Brazil. Food Chem 2007;102:288-294.

25. Castilla-Pinedo Y, Alvis-Estrada L, Alvis-Guzmán N. Exposición a organoclorados por ingesta de leche pasteurizada comercializada en Cartagena, Colombia. Rev Salud Pública 2010;12:14-26.

26. Abou-Arab AAK, Abou DMA, Enb A. Chemical composition, metals content and pesticide residues in raw, pasteurized and UHT milk and their dietary intake. J Egypt Soc Toxicol 2008;39:111-121.

27. Miclean M, Cadar O, Levei EA, Todea DA. Human health risk assessment of organochlorine compounds associated with raw milk consumption in a Romanian industrial area. Ital J Food Sci 2018;30:116-127.

28. INEGI. Instituto Nacional de Estadística, Geografía e Informática. (2009). Prontuario de información geográfica municipal de los Estados Unidos Mexicanos. Jamapa, Veracruz de Ignacio de la Llave. http://www3.inegi.org.mx/contenidos/app/mexicocifras/datos_geograficos/30/30090.p df. Consultado: 13 May, 2018.

29. INEGI. Instituto Nacional de Estadística, Geografía e Informática. (2009). Prontuario de información geográfica municipal de los Estados Unidos Mexicanos. Soledad de Doblado, Veracruz de Ignacio de la Llave. http://www3.inegi.org.mx/contenidos/app/mexicocifras/datos_geograficos/30/30148.p df. Consultado: 13 May, 2018. 
30. Norma Oficial Mexicana NOM-243-SSA1-2010. Productos y servicios. Leche, fórmula láctea, producto lácteo combinado y derivados lácteos. Disposiciones y especificaciones sanitarias. Métodos de prueba. Secretaría de Salud. México, DF. 2010.

31. Alais C. Ciencias de la leche: principio de técnica lechera. 12ª ed. México: CECSA; 1998.

32. Murphy PG. Sulfuric acid cleanup of animal tissues for analysis of acid-stable chlorinated hydrocarbon residues. J AOAC 1972;55:1360-1362.

33. Su GCC. A comparison of statistical and empirical detection limits. J AOAC International 1998;81:105-110.

34. Pandit GG, Sahu SK. Assessment of risk to public health posed by persistent organochlorine pesticide residues in milk and milk products in Mumbai, India. J Environ Monit 2002;4:182-185.

35. Cervera P, Clapes J, Rigolfas R. Alimentación y dietoterapia. $3^{\mathrm{a}}$ ed. Madrid: McGrawHill Interamericana; 1999.

36. INEGI. Instituto Nacional de Estadística, Geografía e Informática. Consumo per cápita de alimentos en México. Mexico. 2002.

37. Thoulon-Page Ch. Cuaderno de dietética: Alimentación de las personas sanas. 2a ed. Barcelona: Masson; 1995.

38. Ziegler EE, Filer LJ. Conocimientos actuales sobre nutrición. Publicación Científica No. 565. 7a ed. Whasington, DC, EUA: Organización Panamericana de la Salud - Instituto Internacional de Ciencias de la Vida; 1997.

39. FAO/WHO. Food and Agriculture Organization and World Health Organization. Guidelines for Predicting Dietary Intake of Pesticide Residues (revised). Global Environment Monitoring System - Food Contamination Monitoring and Assessment Programme (GEMS/Food) in collaboration with the Codex Committee on Pesticide Residues WHO/FSF/FOS/97.7. Switzerland: World Health Organization. 1997.

40. Pardío VT, Waliszewski KN, Landín LA, Bautista RG. Organochlorine pesticide residues in cow's milk from a tropical region of Mexico. Food Addit Contam 2003;20:259-269.

41. Gutiérrez R, Ruíz JL, Ortiz R, Vega S, Schettino B, Yamazaki A, et al. Organochlorine pesticide residues in bovine milk from organic farms in Chiapas, Mexico. Bull Environ Contam Toxicol 2012;89:882-887.

42. ATSDR. Agency for Toxic Substances and Disease Registry. Toxicological profiles of toxic substances. US Department of Health and Human Services, Public Health Service, Atlanta, GA. 2005. http:/www.atsdr.cdc.gov. Accessed: May 13, 2018. 
43. Bajwa U, Singh K. Effect of handling and processing on pesticide residues in food- a review. J Food Sci Technol 2014;51:201-220.

44. EPA. Environmental Protection Agency. Guidelines for carcinogenic risk assessment. Federal Register 51:33992-34003. 1986.

45. Arriaga C, Heredia D, Martínez C, Rayas A. Importancia de los sistemas de producción de leche a pequeña escala en México: ICAR-UAM. México. 2012.

46. Martínez CCJ, Cotera RJ, Zavaleta JA. Características de la producción y comercialización de leche bovina en sistemas de doble propósito en Dobladero Veracruz. Rev Mex Agroneg 2012;16:816-824.

47. FAO/WHO. Food and Agriculture Organization and World Health Organization. Orientaciones para el estudio de las ingestas alimentarias de contaminantes químicos. Ginebra: Organización Mundial de la Salud 1987;87:19-23.

48. Mnif W, Hadj AI, Bouaziz A, Bartegi A, Thomas O, Roig B. Effect of endocrine disruptor pesticides: A review. Int J Environ Res Public Health 2001;8:2265-2303. 